\title{
COLLARS AND PARTITIONS OF HYPERBOLIC CONE-SURFACES
}

\author{
EMILY B. DRYDEN AND HUGO PARLIER
}

\begin{abstract}
For compact Riemann surfaces, the collar theorem and Bers' partition theorem are major tools for working with simple closed geodesics. The main goal of this paper is to prove similar theorems for hyperbolic conesurfaces. Hyperbolic two-dimensional orbifolds are a particular case of such surfaces. We consider all cone angles to be strictly less than $\pi$ to be able to consider partitions.
\end{abstract}

\section{INTRODUCTION}

Compact hyperbolic cone-surfaces are a natural generalization of both compact hyperbolic Riemann surfaces and compact hyperbolic two-dimensional orbifolds. Both the collar theorem and Bers' theorem for partitions are very important tools for Riemann surfaces. It is thus natural to try to find equivalent theorems for two-dimensional orbifolds, and more generally for cone-surfaces.

The collar theorem for compact hyperbolic Riemann surfaces (e.g. [7], [1]) states that surrounding a simple closed geodesic there is a tubular neighborhood, called a collar, which is a topological cylinder. This neighborhood is of a certain width which depends uniquely on the length of the geodesic. Furthermore, if two simple closed geodesics do not intersect then their collars are disjoint. Finally, the values given for the widths of the collars are optimal ([10], [11]). There has been interest in proving a similar theorem for orbifolds (e.g. [4], [6] and [8]), where the object was often to estimate minimal distance between singular points based on the order of the points. The collar theorem for hyperbolic cone-surfaces has the same properties as the original collar theorem, and is thus a natural generalization. Specifically, given a collection of disjoint simple closed geodesics on a hyperbolic cone-surface $M$, we can complete it to a partition of $M$, or a set of pairwise disjoint simple closed geodesics which divide the surface into pairs of pants. We find collars surrounding these simple closed geodesics and the cone points in $M$; the collars are disjoint. The width of the collar about a simple closed geodesic depends on the length of the geodesic and the size of the largest cone angle in the surface, while the width of the collar about a cone point depends on the cone angle at that point. The collars about geodesics are topological cylinders, and those about cone points are topological cones. The values given for the widths of these collars are optimal. The techniques used in the proof are based on hyperbolic trigonometry and both geometric and topological properties of simple closed geodesics.

\footnotetext{
Key words and phrases. Hyperbolic cone-surfaces, Orbifolds, Simple Closed Geodesics, Partitions, Collars.

The first author was partially supported by the U.S. National Science Foundation grant DMS0306752. The second author was supported by the Swiss National Science Foundation grants 21 - 57251.99 and $20-68181.02$.
} 
Bers' partition theorem states that, on a compact hyperbolic Riemann surface, a partition of the surface into pairs of pants can be chosen such that the lengths of the partitioning geodesics are bounded by a constant depending solely on the genus. The optimal constant is not known, although an upper and lower bound are known (e.g. [2]) and certain properties of surfaces that would realize Bers' constant are also known (e.g. [9]). We find a constant analogous to Bers' partition constant that depends on the genus and the number of singular points; any such bound will necessarily depend upon the number of singular points. The techniques used to prove the theorem include area arguments using polar coordinates. An explicit bound is found in the proof.

In the setting of compact hyperbolic Riemann surfaces, Bers' theorem has proved to be a useful tool in studying spectral questions. In particular, it has been used to find a rough fundamental domain for the action of the Teichmüller modular group, to find an explicit bound on the size of isospectral families, and in estimates involving Fenchel-Nielsen parameters (see [2]). Its utility stems from the fact that it allows one to significantly restrict the allowed lengths of partitioning geodesics.

The paper is organized as follows. We begin by giving the necessary background on hyperbolic cone-surfaces. In section 3, we prove the collar theorem (Theorem 3.1) and discuss its consequences. Bers' theorem (Theorem 4.1) is proved in section 4.

The authors are grateful to Peter Buser and Carolyn Gordon for reading and providing feedback on early drafts of this work. Much of this research was done while the first named author was visiting EPFL, and she thanks the geometry group there for their hospitality.

\section{Preliminaries}

Although most results, definitions and properties in this section are readily accepted and known, we have not found all of them in the literature. For this reason, we have tried to be rather complete.

Definition 2.1. A hyperbolic cone-surface is a two-dimensional manifold, $M$, which can be triangulated by hyperbolic geodesic triangles.

We are interested in the compact and orientable case and henceforth will always assume our manifolds to be compact and orientable. Such manifolds can have a certain number of singular points and the set of all such points will be denoted by $\Sigma$. If $\Sigma=\emptyset$ then $M$ is a compact hyperbolic Riemann surface. If $\Sigma \neq \emptyset$ then $M \backslash \Sigma$ has a smooth (although not complete) Riemannian metric with constant curvature -1 . At each singular point $p$, there is a collection of triangles having $p$ as a vertex and with given interior angles at $p$. The sum of these given angles is the cone angle associated to $p$. In what follows, these angles will be considered strictly inferior to $\pi$ for two reasons. First, two singular points with cone angle $\pi$ can be arbitrarily close to each other or to a simple closed geodesic. Thus there can be no natural generalization of the collar theorem from compact hyperbolic Riemann surfaces to hyperbolic cone-surfaces with such singular points. Furthermore, the notion of partition changes significantly when we allow cone angle $\pi$, making it difficult to find a natural generalization of Bers' theorem. If cone angles are all of the form $2 \pi / k$ where $k$ is an integer, then $M$ is an orbifold. Finally, if one lets the 

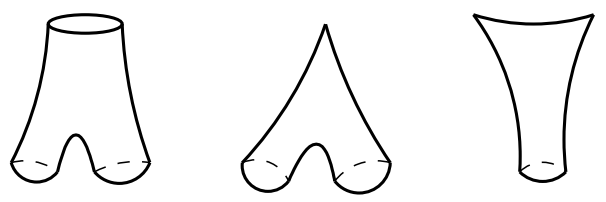

FIGURE 1

cone angles tend towards 0, cusps appear. The case of cusps is well known ([2]) and thus is not mentioned in the theorem. However, our techniques apply to this case as well.

Our objects of study will be called admissible cone-surfaces. These are compact orientable hyperbolic cone-surfaces which satisfy certain conditions. First, all cone angles are less than $\pi$. We also require that $(g, n) \geq(0,4)$, where $g$ is the genus of the surface and $n$ is the number of cone points. Here the ordering is lexicographic ordering; that is, $(g, n) \geq(0,4)$ means that $g \geq 0$, and if $g=0$, then $n \geq 4$. Note that $(g, n)$ cannot equal $(1,0)$, as we require our surfaces to be endowed with a hyperbolic metric.

Two curves are considered freely homotopic if they are freely homotopic on $M \backslash \Sigma$. A closed curve and a cone point $p$ are freely homotopic if they are freely homotopic on $(M \backslash \Sigma) \cup\{p\}$. Furthermore, by curve or geodesic we mean the set of all points lying on the curve. The following properties for simple closed curves are a result of the constant negative curvature, and can be proved in an identical fashion to their equivalent for Riemann surfaces.

Proposition 2.2. Let $M$ be an admissible cone-surface.

(1) Every non-trivial simple closed curve on $M \backslash \Sigma$ is freely homotopic to either a unique simple closed geodesic or a unique cone point. (For a closed curve $\delta$, the associated closed geodesic will be denoted $\mathcal{G}(\delta)$.)

(2) If two non-trivial closed curves $\alpha$ and $\beta$ intersect $n$ times, then $\mathcal{G}(\alpha)$ and $\mathcal{G}(\beta)$ intersect at most $n$ times, or $\mathcal{G}(\alpha)=\mathcal{G}(\beta)$.

(3) Given two non-intersecting smooth simple curves $\alpha$ and $\beta$ on $M$ there is at least one geodesic path $c$ between them such that $d_{M}(\alpha, \beta)$ is realized by c. Such a path $c$ is perpendicular to $\alpha$ and $\beta$. If $\alpha$ and $\beta$ are geodesic, in a free homotopy class of paths with end points moving on $\alpha$ and $\beta$, such a path $c$ is unique. This property remains true for singular points in place of one or both geodesics.

Based on properties in the previous proposition, one can define a partition to be a set of simple closed geodesics $\mathcal{P}=\left\{\gamma_{1}, \ldots, \gamma_{m}\right\}$ such that $M \backslash \mathcal{P}$ is a set of pairs of pants. A pair of pants is defined as for smooth surfaces, except a boundary geodesic can be replaced by a cone point. We have $m=3 g-3+n$ where $g$ is the genus and $n$ the number of singular points. If a pair of pants has three geodesics as boundary, then it will be called a $Y$-piece. If it has one cone point and two geodesics as boundary then it will be called a $V$-piece. Finally, if the pair of pants has two cone points and one geodesic as boundary then it will be called a joker's hat. See Figure 1 for examples of the various types of pairs of pants, and Figure 2 for an example of a partition. 


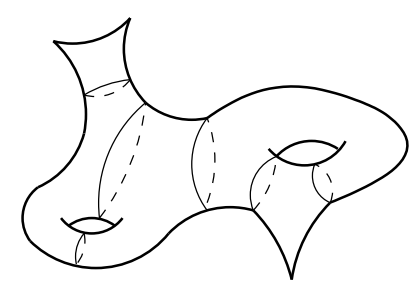

Figure 2

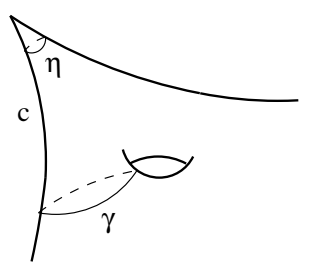

FiguRE 3

The only situation in which a pair of pants has three cone points as boundary is if $M$ is a sphere with three cone points. For other basic properties of hyperbolic cone-surfaces see [3].

It is well known that a $Y$-piece can be decomposed into two isometric rightangled hexagons in the hyperbolic plane. In the same fashion, a $V$-piece can be decomposed into two isometric pentagons with four right angles ([4]), and a joker's hat into two isometric quadrilaterals with two right angles. Both such hyperbolic pentagons and quadrilaterals can in turn be broken down into two (not necessarily isometric) trirectangles, which are quadrilaterals with three right angles. For hyperbolic formulas for these polygons, see [2] or [5].

\section{The Collar theorem}

Let $p$ be a cone point and $\gamma$ a simple closed geodesic on $M$. Let $c$ be a simple geodesic path from $p$ to $\gamma$, perpendicular to $\gamma$. These elements describe a unique pair of pants in the following manner. Let $\delta$ be the closed curve obtained by taking $\gamma \circ c \circ \eta \circ c^{-1}$ as in Figure 3.

Then $\mathcal{G}(\delta)$ is either a simple closed geodesic or another cone point, and in either case $(p, \gamma, \mathcal{G}(\gamma))$ is a pair of pants. Using exactly the same technique with two cone points and a simple geodesic path between them, or with two simple closed geodesics and a perpendicular simple geodesic path between them, we get pairs of pants that are uniquely determined. This construction is essential for the following theorem.

Theorem 3.1. Let $M$ be an admissible cone-surface of genus $g$ with $n$ cone points $p_{1}, \ldots, p_{n}$ with cone angles $2 \varphi_{1}, \ldots, 2 \varphi_{n}$. Let $2 \varphi$ be the largest cone angle. Let $\gamma_{1}, \ldots, \gamma_{m}$ be disjoint simple closed geodesics on $M$. Then the following hold.

(1) $m \leq 3 g-3+n$. 

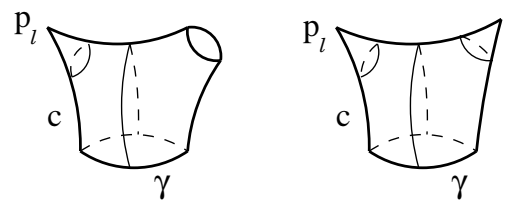

Figure 4

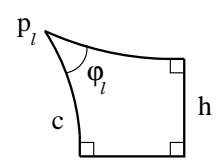

FiguRE 5

(2) There exist simple closed geodesics $\gamma_{m+1}, \ldots, \gamma_{3 g-3+n}$ which together with $\gamma_{1}, \ldots, \gamma_{m}$ form a partition of $M$.

(3) The collars

$$
\mathcal{C}\left(\gamma_{k}\right)=\left\{x \in M \mid d\left(x, \gamma_{k}\right) \leq w_{k}=\operatorname{arcsinh}\left(\cos \varphi / \sinh \frac{\gamma_{k}}{2}\right)\right\}
$$

and

$$
\mathcal{C}\left(p_{l}\right)=\left\{x \in M \mid d\left(x, p_{l}\right) \leq v_{l}=\operatorname{arccosh}\left(1 / \sin \varphi_{l}\right)\right\}
$$

are pairwise disjoint for $k=1, \ldots, 3 g-3+n$ and $l=1, \ldots, n$.

(4) Each $\mathcal{C}\left(\gamma_{k}\right)$ is isometric to the cylinder $\left[-w_{k}, w_{k}\right] \times \mathbb{S}^{1}$ with the Riemannian metric $d s^{2}=d \rho^{2}+\ell^{2}\left(\gamma_{k}\right) \cosh ^{2} \rho d t^{2}$.

Each $\mathcal{C}\left(p_{l}\right)$ is isometric to a hyperbolic cone $\left[0, v_{l}\right] \times \mathbb{S}^{1}$ with the Riemannian metric $d s^{2}=d \rho^{2}+\frac{\varphi_{l}^{2}}{\pi^{2}} \sinh ^{2} \rho d t^{2}$.

Proof. The first two points are equivalent to the problem of counting the number of geodesics in a partition for a surface of signature $(g, n)$, and showing that any collection of pairwise disjoint simple closed geodesics can be completed to form a partition. These questions are not new and the proofs are known (e.g. [2]).

The first step in proving the theorem is to show that a cone point $p_{l}$ is at a distance of at least $\operatorname{arccosh}\left(1 / \sin \varphi_{l}\right)$ from all simple closed geodesics. Let $\gamma$ be a simple closed geodesic on $M$. Take a geodesic path $c$ that realizes the distance between $p_{l}$ and $\gamma$ (i.e. such that $\ell(c)=d\left(p_{l}, \gamma\right)$ ). Then take the unique pair of pants obtained from $p_{l}, \gamma$ and $\mathcal{G}\left(\gamma \circ c \circ \eta \circ c^{-1}\right)$ as discussed previously. Either this pair of pants is a $V$-piece or a joker's hat. In both cases extract the trirectangle (a hyperbolic quadrilateral with three right angles) as in Figures 4 and 5.

From the formulas for the trirectangle we have

$$
\cosh c=\frac{\cosh h}{\sin \varphi_{l}}
$$

where $h$ is as in Figure 5. It follows that $c>\operatorname{arccosh}\left(1 / \sin \varphi_{l}\right)$. 


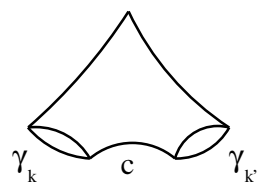

FiguRE 6

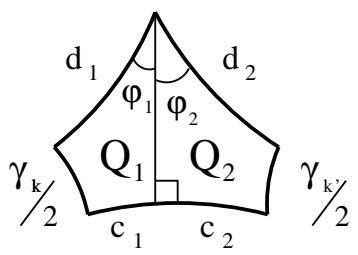

FiguRe 7

Let $p_{l}$ and $p_{l^{\prime}}$ be two distinct cone points. Let $b$ be a path that realizes distance between them. Let $\gamma$ be a simple closed geodesic that crosses $b$. It is clear that $\ell(b)$ is necessarily greater than or equal to the distance from $p_{l}$ to $\gamma$ added to the distance from $p_{l^{\prime}}$ to $\gamma$. From what precedes we have:

$$
\ell(b) \geq d\left(p_{l}, \gamma\right)+d\left(p_{l^{\prime}}, \gamma\right)>\operatorname{arccosh}\left(\frac{1}{\sin \varphi_{l}}\right)+\operatorname{arccosh}\left(\frac{1}{\sin \varphi_{l^{\prime}}}\right) .
$$

It follows that the distance sets $\mathcal{C}\left(p_{l}\right)$ and $\mathcal{C}\left(p_{l^{\prime}}\right)$ are disjoint.

Let $\gamma_{k}$ and $\gamma_{k^{\prime}}$ be two disjoint simple closed geodesics. Let $c$ be a path that realizes distance between them. The unique pair of pants determined by $c, \gamma_{k}$ and $\gamma_{k^{\prime}}$ is either a $Y$-piece or a $V$-piece. In the first case it follows that $\mathcal{C}\left(\gamma_{k}\right)$ and $\mathcal{C}\left(\gamma_{k^{\prime}}\right)$ are disjoint sets from the collar theorem on Riemann surfaces. In the latter case, consider Figures 6 and 7.

Notice that only half of the collar around a given boundary geodesic is contained in a pair of pants. Also note that both angles $\varphi_{1}$ and $\varphi_{2}$ are strictly inferior to $\varphi$. From the trirectangle formula applied to $Q_{1}$ we obtain

$$
\sinh c_{1}=\frac{\cos \varphi_{1}}{\sinh \frac{\gamma_{k}}{2}}
$$

and analogously for $Q_{2}$. From this we obtain that

and

$$
c_{1}=\operatorname{arcsinh}\left(\frac{\cos \varphi_{1}}{\sinh \frac{\gamma_{k}}{2}}\right)
$$

$$
c_{2}=\operatorname{arcsinh}\left(\frac{\cos \varphi_{2}}{\sinh \frac{\gamma_{k^{\prime}}}{2}}\right) .
$$

It follows that the distance sets $\mathcal{C}\left(\gamma_{k}\right)$ and $\mathcal{C}\left(\gamma_{k^{\prime}}\right)$ are disjoint, because each collar is the union of two such half-collars.

It remains to prove that for arbitrary $p_{l}$ and $\gamma_{k}$ the collars are disjoint. Let $c$ be a geodesic path that realizes the distance between them. The collars around both the cone point and the geodesic have widths which depend only on their angle or length. It thus suffices to consider the case where the cone point and the geodesic 


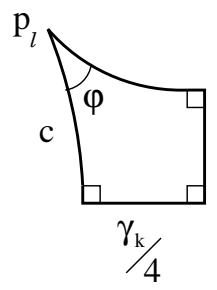

FiguRE 8

would be as close as possible. This case would occur if the pair of pants resulting from $p_{l}, \gamma_{k}$ and $c$ were a joker's hat with both cone angles equal to $2 \varphi$. (This is a direct consequence of hyperbolic geometry.) In this case Figure 8 would apply.

From a trirectangle formula one obtains

$$
\sinh c=\operatorname{coth} \frac{\gamma_{k}}{4} \cot \varphi
$$

This in turn can be expressed as

$$
\sinh c=\frac{\cosh \frac{\gamma_{k}}{2}+1}{\sinh \frac{\gamma_{k}}{2}} \cot \varphi .
$$

Let us compare this with the value obtained by calculating $\sinh \left(w_{k}+v_{l}\right)$. By calculation one obtains

$$
\sinh \left(w_{k}+v_{l}\right)=\frac{1+\sqrt{\cos ^{2} \varphi+\sinh ^{2} \frac{\gamma_{k}}{2}}}{\sinh \frac{\gamma_{k}}{2}} \cdot \cot \varphi .
$$

By comparison and because $\varphi<\frac{\pi}{2}$ we obtain that $c>w_{k}+v_{l}$. This implies that the distance sets $\mathcal{C}\left(\gamma_{k}\right)$ and $\mathcal{C}\left(p_{l}\right)$ are disjoint.

The last point of the theorem is obtained as in the classical theory of Riemann surfaces for $\mathcal{C}\left(\gamma_{k}\right)$ (see [2]), and was shown by Dianu [4] for $\mathcal{C}\left(p_{l}\right)$.

Remark 3.2. The values for the collars are in general optimal in the following sense. The collar around a simple closed geodesic $\gamma$ can be seen as a distance set with the following property: if another simple closed geodesic enters $\mathcal{C}(\gamma)$, then it necessarily intersects $\gamma$. Suppose $\gamma$ is a non-separating simple closed geodesic on an admissible cone-surface with $(g, n) \neq(1,1)$. (If $(g, n)=(1,1)$ then all simple closed geodesics intersect.) Now replacing $w_{\gamma}=\operatorname{arcsinh}\left(\cos \varphi / \sinh \frac{\gamma}{2}\right)$ by $w=w_{\gamma}+\varepsilon$ with $\varepsilon>0$ would be fatal to this intersection property. In fact, for any $\varepsilon>0$ there are an infinity of simple closed geodesics that intersect the enlarged collar but not $\gamma$. To prove this, take a $V$-piece containing $\gamma$ and $p$ where the cone angle at $p$ is exactly $2 \varphi$. The other boundary geodesic $\gamma^{\prime}$ of the $V$-piece can be chosen as long as desired (see [10]). From the formulas obtained in the proof, it follows that $\gamma^{\prime}$ can be arbitrarily close to the collar of $\gamma$. In the case where $\gamma$ is a separating geodesic, the optimal collar width will depend on how the cone angles are distributed on both sides of $\gamma$; using the same techniques, one could find an optimal collar in each of the various cases.

In an analogous fashion, one can show that the bound for collars around cone points is also sharp, provided one can include the cone point in a $V$-piece with two distinct simple closed geodesics. This excludes only the cases where $(g, n)=(1,1)$ 
and $(0,4)$. In the latter case, the optimal collar width depends on all four cone angles. Now if the surface is a torus $T$ with a single cone point $p$ with cone angle $2 \varphi$, by cutting along an interior simple closed geodesic of $T$, say $\gamma$, one obtains a $V$-piece with two boundary geodesics of equal length. The process of lengthening only one of the two geodesics is no longer possible and this extra rigidity implies that the sharp constant is in fact $\operatorname{arccosh}\left(1 / \sin \frac{\varphi}{2}\right)$ (cf. [10]).

An example of the utility of the collar theorem is the following natural corollary.

Corollary 3.3. Let $\gamma$ and $\delta$ be closed geodesics on $M$ which intersect each other transversally, and assume that $\gamma$ is simple. Then

$$
\sinh \frac{\ell(\gamma)}{2} \sinh \frac{\ell(\delta)}{2}>\cos \varphi
$$

\section{Partitions}

We now proceed to prove Bers' theorem for compact admissible cone-surfaces.

Theorem 4.1. Let $M$ be an admissible cone-surface of genus $g$ with $n$ cone points. Then there exists a partition $\mathcal{P}$ of $M$ such that every geodesic in $\mathcal{P}$ has length less than a constant $L_{g, n}$.

Proof. Let $p_{1}, \ldots, p_{n}$ be the cone points on $M$ with corresponding cone angles $2 \varphi_{1}, \ldots, 2 \varphi_{n}$. We define

$$
Z_{i}\left(r_{i}\right)=\left\{x \in M \mid \operatorname{dist}\left(x, p_{i}\right) \leq r_{i}\right\},
$$

for $i=1, \ldots, n$. We denote the boundary of $Z_{i}\left(r_{i}\right)$ by $\beta_{i}$. Each neighborhood $Z_{i}\left(r_{i}\right)$ admits polar coordinates, and we have

$$
\text { Area } Z_{i}\left(r_{i}\right)=2 \varphi_{i}\left(\cosh r_{i}-1\right) \text {. }
$$

For $i=1, \ldots, n$, we also know that

$$
\text { Area } Z_{i}\left(r_{i}\right)<\text { Area } M \text {. }
$$

This implies

$$
r_{i}<\operatorname{arccosh}\left(1+\frac{\text { Area } M}{2 \varphi_{i}}\right)
$$

Now

$$
\ell\left(\beta_{i}\right)=2 \varphi_{i} \sinh r_{i}
$$

SO

$$
\begin{aligned}
\ell\left(\beta_{i}\right) & <2 \varphi_{i} \sinh \left(\operatorname{arccosh}\left(1+\frac{\operatorname{Area} M}{2 \varphi_{i}}\right)\right) \\
& =2 \varphi_{i} \sqrt{\left(1+\frac{\text { Area } M}{2 \varphi_{i}}\right)^{2}-1} \\
& <\text { Area } M+2 \varphi_{i} \\
& <2 \pi(2 g-2+n) .
\end{aligned}
$$

Thus the length of the boundary of the neighborhood $Z_{i}\left(r_{i}\right)$ about $p_{i}, i=1, \ldots, n$, is bounded above by $2 \pi(2 g-2+n)$.

Starting from $r_{i}=0$, we let $r_{i}$ grow continuously (for all $i$ simultaneously) until one of the following cases occurs:

(1) $\beta_{j}$ ceases to be simple for some $j \in\{1, \ldots, n\}$; 


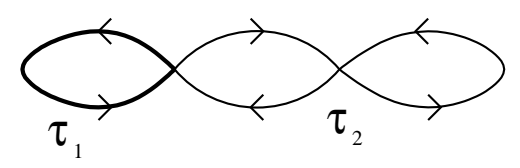

FiguRE 9

(2) $\beta_{j}$ meets $\beta_{k}$ for some $j \neq k$.

Once one of these cases occurs, we fix all the $r_{i}$. We will consider each case in turn.

Case 1. We view $\beta_{j}$ as the composition of two curves $\tau_{1}$ and $\tau_{2}$, which both have initial and final points at the self-intersection of $\beta_{j}$. If $\beta_{j}$ has multiple selfintersections, then we choose one and let $\tau_{1}$ and $\tau_{2}$ be as in Figure 9 .

We allow $\tau_{1}$ and $\tau_{2}$ to slide in their free homotopy classes on $M \backslash \Sigma$ until they reach their minima. We have

$$
\ell\left(\mathcal{G}\left(\tau_{1}\right)\right)<\ell\left(\tau_{1}\right)<\ell\left(\beta_{j}\right)<2 \pi(2 g-2+n),
$$

and similarly for $\ell\left(\mathcal{G}\left(\tau_{2}\right)\right)$. Note that it is possible that one of $\mathcal{G}\left(\tau_{1}\right)$ or $\mathcal{G}\left(\tau_{2}\right)$ is a cone point (but not both, as $M$ has signature $(g, n) \geq(0,4)$ ), in which case the nontrivial geodesic bounds a joker's hat. We cut $M$ open along those $\mathcal{G}\left(\tau_{i}\right), i=1,2$, which are not cone points, and remove any resulting $V$-pieces or joker's hats. Let $M^{1}$ be the (possibly empty) remaining connected component. Then

$$
\ell\left(\partial M^{1}\right)<4 \pi(2 g-2+n) .
$$

Case 2. Suppose that two boundary curves $\beta_{j}$ and $\beta_{k}$ meet, and that both $\beta_{j}$ and $\beta_{k}$ are simple (if more than two simple boundary curves meet, choose two). Consider the curve $\tau$ obtained by first traversing $\beta_{j}$ and then traversing $\beta_{k}$, where the initial and final points of both of these curves are at their intersection point. Note that $\tau$ is homotopic to a simple closed curve and is not homotopic to a cone point, as $M$ has signature $(g, n) \neq(0,3)$. Thus, $\tau$ is homotopic to a unique simple closed geodesic. We have

$$
\ell(\mathcal{G}(\tau))<\ell(\tau)=\ell\left(\beta_{j}\right)+\ell\left(\beta_{k}\right)<4 \pi(2 g-2+n) .
$$

Cutting $M$ open along this geodesic yields at least one joker's hat. Let $M^{1}$ denote the (cone-)surface obtained by cutting $M$ open in this way and removing any joker's hats that result. Then

$$
\ell\left(\partial M^{1}\right)<4 \pi(2 g-2+n) .
$$

We now restart the process; that is, we send out collars $Z_{i}\left(r_{i}\right)$ from the remaining cone points $p_{i}$ on $M^{1}$ and let $r_{i}$ grow continuously from $r_{i}=0$ until one of the following situations occurs:

(1) $\beta_{j}$ ceases to be simple for some $j \in\{1, \ldots, n\}$;

(2) $\beta_{j}$ meets $\beta_{k}$ for some $j \neq k$;

(3) $\beta_{j}$ meets a boundary geodesic $\gamma_{i}$ on $M^{1}$.

Once one of these cases occurs, we fix all the $r_{i}$. Cases 1 and 2 are as above, and if we let $M^{2}$ be the (cone-)surface which results from cutting $M^{1}$ open along the 
new geodesics we find and removing all Y-pieces, V-pieces and joker's hats, then

$$
\ell\left(\partial M^{2}\right)<8 \pi(2 g-2+n) .
$$

Case 3. Consider the curve $\tau$ obtained by first traversing $\beta_{j}$ and then traversing $\gamma_{i}$, where the initial and final points of both of these curves are at their intersection point. Note that $\tau$ is homotopic to a simple curve and is not homotopic to a cone point; if it were, then $p_{j}$ would live on a joker's hat that would have been removed at the previous step. Thus, $\tau$ is homotopic to a unique simple closed geodesic. We have

$\ell(\mathcal{G}(\tau))<\ell(\tau)=\ell\left(\beta_{j}\right)+\ell\left(\gamma_{i}\right)<2 \pi(2 g-2+n)+4 \pi(2 g-2+n)=6 \pi(2 g-2+n)$.

Cutting $M^{1}$ open along $\mathcal{G}(\tau)$ yields at least one $\mathrm{V}$-piece. Let $M^{2}$ be as defined above; then

$$
\ell\left(\partial M^{2}\right)<\ell\left(\partial M^{1}\right)+2 \pi(2 g-2+n)<6 \pi(2 g-2+n),
$$

as $\ell(\mathcal{G}(\tau))<\ell\left(\gamma_{i}\right)+2 \pi(2 g-2+n)$ and $\gamma_{i} \notin \partial M^{2}$.

We repeat the above process until all of the cone points on $M$ have been removed on V-pieces or joker's hats. Note that at each step, after cutting our cone-surface open along the geodesics we find and removing any Y-pieces, V-pieces and joker's hats, the length of the boundary of the resulting (cone-)surface increases by at most $4 \pi(2 g-2+n)$. To remove all $n$ cone points requires $m \leq 2 n$ geodesics and $\mu \leq m$ steps; thus, we have found $\gamma_{1}, \ldots, \gamma_{m}$ such that

$$
\ell\left(\partial M^{j}\right)<4 \pi j(2 g-2+n), \quad j=1, \ldots, \mu
$$

and

$$
\ell\left(\gamma_{k}\right)<4 \pi k(2 g-2+n), \quad k=1, \ldots, m .
$$

To obtain the remaining geodesics in our decomposition, we proceed by induction. That is, we find a suitable simple closed geodesic in the interior of $M^{\mu}$ which is not homotopic to a boundary component, cut $M^{\mu}$ open along this geodesic, remove any $Y$-pieces, and let $M^{\mu+1}$ be the resulting surface. To find such a geodesic, we create tubular neighborhoods around all boundary geodesics and let the widths grow until a critical case occurs; the area arguments are analogous to those in the induction for compact Riemann surfaces of genus $g \geq 2$ (see [2]).

Remark 4.2. The proof gives an explicit bound for the length of each partitioning geodesic:

$$
\ell\left(\gamma_{k}\right)<4 \pi k(2 g-2+n)
$$

where $\gamma_{k}$ is the $k$ th geodesic in a partition of $M$. For $L_{g, n}$ we have thus proved the following bound:

$$
L_{g, n}<4 \pi(3 g-3+n)(2 g-2+n) .
$$

\section{REFERENCES}

[1] Peter Buser. The collar theorem and examples. Manuscripta Math., 25(4):349-357, 1978.

[2] Peter Buser. Geometry and spectra of compact Riemann surfaces, volume 106 of Progress in Mathematics. Birkhäuser Boston Inc., Boston, MA, 1992.

[3] Daryl Cooper, Craig D. Hodgson, and Steven P. Kerckhoff. Three-dimensional orbifolds and cone-manifolds, volume 5 of MSJ Memoirs. Mathematical Society of Japan, Tokyo, 2000. With a postface by Sadayoshi Kojima. 
[4] Rares Dianu. Sur le spectre des tores pointés. PhD thesis, Ecole Polytechnique Fédérale de Lausanne, 2000.

[5] Werner Fenchel and Jakob Nielsen. Discontinuous groups of isometries in the hyperbolic plane, volume 29 of de Gruyter Studies in Mathematics. Walter de Gruyter \& Co., Berlin, 2003. Edited and with a preface by Asmus L. Schmidt, Biography of the authors by Bent Fuglede.

[6] F. W. Gehring and G. J. Martin. Hyperbolic axes and elliptic fixed points in a Fuchsian group. Complex Variables Theory Appl., 33(1-4):303-309, 1997.

[7] Linda Keen. Collars on Riemann surfaces. In Discontinuous groups and Riemann surfaces (Proc. Conf., Univ. Maryland, College Park, Md., 1973), pages 263-268. Ann. of Math. Studies, No. 79. Princeton Univ. Press, Princeton, N.J., 1974.

[8] J. Peter Matelski. A compactness theorem for Fuchsian groups of the second kind. Duke Math. J., 43(4):829-840, 1976.

[9] Hugo Parlier. Lengths of geodesics on Riemann surfaces with boundary. Ann. Acad. Sci. Fenn. Math., 30:227-236, 2005.

[10] Hugo Parlier. A note on collars of simple closed geodesics. Geom. Dedicata, 112:165-168, 2005.

[11] Mika Seppälä and Tuomas Sorvali. Geometry of Riemann surfaces and Teichmüller spaces, volume 169 of North-Holland Mathematics Studies. North-Holland Publishing Co., Amsterdam, 1992.

Department of Mathematics, Bucknell University, Lewisburg, PA 17837

E-mail address: ed012@bucknell.edu

Section de Mathématiques, Université de Genève, Switzerland

E-mail address: hugo.parlier@math.unige.ch 